INPLASY

PROTOCOL

To cite: Wang et al.

Acupuncture therapy for Gastric ulcer (Protocol for a systematic review and metaanalysis). Inplasy protocol 202130085. doi:

10.37766/inplasy2021.3.0085

Received: 22 March 2021

Published: 23 March 2021

Corresponding author:

Heran Wang

dr824775122@163.com

Author Affiliation:

Changchun University of

Traditional Chinese Medicine

Support: NSFC: 82004472 ; 81973954.

Review Stage at time of this submission: The review has not yet started.

Conflicts of interest:

None declared.

\section{Acupuncture therapy for Gastric ulcer (Protocol for a systematic review and meta-analysis)}

Wang, $\mathrm{H}^{1}$; Jiang, $\mathrm{H}^{2}$; Zhao, J3; Han, Y4; Zhao, $\mathrm{X}^{5}$; Meng, M6; Pan, T7; Li, T8; Wang, F9.

Review question / Objective: The protocol of this study will systematically assess the effectiveness and safety of acupuncture for patients with Gastric ulcer.

Condition being studied: Gastric ulcer (GU), a common digestive disease, has a high incidence and seriously endangers health of human. Gastric ulcer is a chronic disease affecting up to $10 \%$ of the world's population. The estimated prevalence of peptic ulcer disease in the general population is 5-10\%. According to the previous studies, it has been proved that acupuncture at acupoints had a good effect on GU.

Information sources: The main sources of information that will be obtained in this study include electronic resource databases, The following electronic databases will be searched from the respective dates of database inception to March 23st, 2021. We plan to search eight English and Chinese electronic databases, including the Web of Science, Cochrane Library, PubMed, EMBASE, SinoMed, Wanfang, China Science and Technology Journal (VIP), and China National Knowledge Infrastructure (CNKI) databases, for potentially eligible studies. We will also search dissertations, conference proceedings, and reference lists of relevant included studies.

INPLASY registration number: This protocol was registered with the International Platform of Registered Systematic Review and Meta-Analysis Protocols (INPLASY) on 23 March 2021 and was last updated on 23 March 2021 (registration number INPLASY202130085).

\section{INTRODUCTION}

Review question / Objective: The protocol of this study will systematically assess the effectiveness and safety of acupuncture for patients with Gastric ulcer.
Condition being studied: Gastric ulcer (GU), a common digestive disease, has a high incidence and seriously endangers health of human. Gastric ulcer is a chronic disease affecting up to $10 \%$ of the world's population. The estimated prevalence of 
peptic ulcer disease in the general population is $5-10 \%$. According to the previous studies, it has been proved that acupuncture at acupoints had a good effect on GU.

\section{METHODS}

Search strategy: The following electronic databases will be searched from the respective dates of database inception to March 23st, 2021: The Cochrane Library, Web of Science, EMBASE, MEDLINE, China National Knowledge Infrastructure(CNKI), the Chinese Biomedical Literature Database(CBM), Wanfang database, the Chinese Scientific Journal Database(VIP), and other sources. Randomized controlled trials comparing acupuncture with other interventions or sham acupuncture will be included. Two independent researchers will operate article retrieval, duplication removing, screening, quality evaluation, and data analyses by Review Manager (V.5.3.5). Meta-analyses, subgroup analysis and/or descriptive analysis will be performed based on the included data conditions.

Participant or population: Patients who were diagnosed as Gastric ulcer, will be included, without limits on gender, race, nationality, and medical units.

Intervention: Interventions can be any type of acupuncture.

Comparator: Multiple control interventions will be included: no treatment, placebo and other interventions (eg, cupping therapy, drugs, and physical interventions, moxibustion). If its interventions and comparisons both contain acupuncture, the study will be excluded. Interventions of acupuncture combined with other therapies will be included, only if these combinations are compared to the other therapies semplice.

Study designs to be included: All randomized controlled trials (RCTs) of the application of acupuncture in the treatment of patients with Gastric ulcer will be included with no language limitation.
However, animal studies, case reports, case series, commentaries, reviews, noncontrolled trials, and non-RCTs will be excluded. Randomized controlled trial (RCT) and blinded research will be included. Published clinical trials that reported the efficacy and safety on Acupuncture for Gastric ulcer will be included.

Eligibility criteria: Interventions can be any type of acupuncture, Multiple control interventions will be included: no treatment, placebo and other interventions (eg, cupping therapy, drugs, and physical interventions, moxibustion). If its interventions and comparisons both contain acupuncture, the study will be excluded. Interventions of acupuncture combined with other therapies will be included, only if these combinations are compared to the other therapies semplice.

Information sources: The main sources of information that will be obtained in this study include electronic resource databases, The following electronic databases will be searched from the respective dates of database inception to March 23st, 2021. We plan to search eight English and Chinese electronic databases, including the Web of Science, Cochrane Library, PubMed, EMBASE, SinoMed, Wanfang, China Science and Technology Journal (VIP), and China National Knowledge Infrastructure (CNKI) databases, for potentially eligible studies. We will also search dissertations, conference proceedings, and reference lists of relevant included studies.

Main outcome(s): The protocol of this study will systematically assess the effectiveness and safety of acupuncture for patients with Gastric ulcer. The primary outcome is effective rate.

Additional outcome(s): The secondary outcomes include negative conversing rate of hp, untoward effect, recurrence rate, quality of life. 
Data management: Two independent authors (HYR and MM) will data from the selected eligible articles entered into an Excel form. The extracted information includes the reference ID, name of the first author, time of publication, country, participant characteristics, intervention, sample size, blinding, randomization, outcome measures, duration of follow-up, adverse effects, and other detailed information. If necessary, we will contact the corresponding authors of trials as much as possible for further information.

Quality assessment / Risk of bias analysis: Two review authors will independently evaluate the risk of bias to evaluate the quality of the studies using the Cochrane Collaborationi's risk-of-bias assessment method. The risk of bias will be assessed and classified according to 3 levels: low risk, unclear risk, and high risk. Any discrepancies will be resolved through discussions and negotiations with the third author. When a consensus on the risk assessment cannot be reached by discussion, the third reviewer will make the decision.

Strategy of data synthesis: A meta-analysis or descriptive analysis will be carried out based on measurement methods, intervention methods, heterogeneity levels, etc. If clinical and methodological heterogeneity are low, the fixed-effect model will be applied by merger analysis; the random-effects model will be applied by merger analysis when heterogeneity indicates a moderate level. If a significant level of heterogeneity is found, a descriptive analysis will be performed instead.

Subgroup analysis: Subgroup analysis will be performed based on the findings from the data synthesis. If the heterogeneity is found to have been caused by particular features of the included studies (eg, the intervention methods [type, time, and cycle] and the measurement methods used in the clinical trials), subgroup analysis will be conducted relevant to these categories.
Sensitivity analysis: We will conduct a sensitivity analysis to identify whether the conclusions are robust in the review according to the following criteria: sample size, heterogeneity qualities, and statistical model (random-effects or fixed-effects model).

Country(ies) involved: China.

Keywords: protocol; systematic; Gastric ulcer; Acupuncture.

Contributions of each author:

Author 1 - Heran Wang.

Author 2 - Hailin Jiang.

Author 3 - Jinying Zhao.

Author 4 - Yiran Han.

Author 5 - Xuewei Zhao.

Author 6 - Meng Meng.

Author 7 - Ting Pan.

Author 8 - Tie Li.

Author 9 - Fuchun Wang. 